\title{
Constraint equation for the lowest Landau level in the fractional quantum Hall system
}

\author{
B. Sakita \\ Department of Physics, City College of the City University of New York, New York, New York 10031 \\ Dong-Ning Sheng and Zhao-Bin Su \\ Institute of Theoretical Physics, Academia Sinica, P.O. Box 2735, Beijing, China \\ and Texas Center for Superconductivity at University of Houston, Houston, Texas 77204
}

(Received 9 April 1991; revised manuscript received 24 May 1991)

\begin{abstract}
We apply the singular gauge transformation to the constraint equation for the lowest Landau level to investigate the generic properties of the quasiparticles of the fractional quantum Hall system by using the collective-field-theory approach. It shows a transparent connection with Laughlin's wave functions. If we take an average over the wave functional for the constraint equation, the resulting equation can be interpreted as the vortex equation for the fractionally charged quasiparticles. By introducing a generalized duality transformation, $\rho$ (density)- $\vartheta$ (phase) transformation, we can obtain the fractional statistics as well as the hierarchy scheme from the constraint equation.
\end{abstract}

At present we have a fairly good understanding of the fractional quantum Hall effect ${ }^{1}$ (FQHE) based on Laughlin's wave-function approach. ${ }^{2}$ Stimulated by an investigation of the off-diagonal long-range order for the FQHE, Girvin, and Girvin and MacDonald ${ }^{3}$ and others $^{4-6}$ proposed a Ginzburg-Landau-like effective-field theory of the FQHE. This is in fact an effective-field theory of a $(2+1)$-dimensional Schrödinger-Bose wave field coupled with a Chern-Simons gauge field that exhibits vortex solutions with finite energy and fractional charge. Although these are appealing, their connection to Laughlin's wave-function approach is not entirely clear. In particular, it is still not very clear to what extent the lowest Landau level (LLL) constraint has been properly accounted for.

At the lowest Landau level the kinetic-energy part of the electron Hamiltonian, which usually bears the gauge properties of the system, should be projected away with only the Coulomb interaction term left. Therefore it is reasonable to expect that a rich variety of interesting properties for the FQHE systems, which are independent of the concrete form of the interactions, can be explored via the application of the Chern-Simons gauge-fieldtheory approach to the constraint for the electrons in the LLL. In this paper, we express the condition that all the electrons are in the LLL as a constraint equation for the state functional and apply the collective-field-theory technique ${ }^{7,8}$ which in fact is a field-theoretic approach, to this equation. The general solution of this constraint equation shows a transparent connection to Laughlin's wave function. If we take a quantum average of the constraint equation, the resulting equation can be interpreted as a vortex equation for the fractionally charged quasiparticles that is derived directly from the microscopic theory. The Coulomb interaction does not appear explicitly in the equation. The vortex solutions in turn require singularities of the phase of the wave functional. In the particle coordinate representation these singularities are pre. cisely the ones that appear in the singular exponent of the prefactor of Laughlin's quasiparticles wave function. Next we introduce a generalized duality transformation as a generalized Fourier transformation- $\rho$ (electron density) $\vartheta$ (phase variable conjugate to the electron density) transformation-of the state functional. Then the transformed constraint equation not only makes the fractional charge and statistics of quasiparticles (vortices) explicit, but also provides a natural derivation for the hierarchy scheme. ${ }^{9}$

We introduce the Hamiltonian for the interacting $N$ electron system in a strong uniform magnetic field $B$ by subtracting the zero-point energy for the lowest Landau level $N \hbar e B / 2 m_{0} c$ as

$$
H=\frac{1}{2 m_{0}} \sum_{i}\left(\Pi_{i}^{x}+i \Pi_{i}^{y}\right)\left(\Pi_{i}^{x}-i \Pi_{i}^{y}\right)+V_{c},
$$

where $\mathbf{r}_{i}$ is the two-dimensional coordinate for the $i$ th electron, the sum is taken over all electrons $i=1, \ldots, N$, and $m_{0}$ is the electron mass. In Eq. (1), $\Pi=-i \hbar \nabla+e \mathbf{A}(\mathbf{r}) / c$ and $V_{c}$ is the Coulomb interaction involving both electrons and the background.

There are two energy scales with this system, the Landau energy $\hbar e B / m_{0} c$ and the Coulomb interaction energy $e^{2} / \lambda$, with $\lambda$ being the inverse of the classical cyclotron radius $\lambda=(\hbar c / e B)^{1 / 2}$. In the FQHE regime, however, the former is much larger than the latter and one may assume that all the electrons are in the lowest Landau level. Accordingly, $e^{2} / \lambda$ is the only relevant energy scale. Effectively one may set $m_{0} \rightarrow 0$. Thus, from Hamiltonian (1) and the Schrödinger equation we obtain

$$
\left(\Pi_{i}^{x}-i \Pi_{i}^{y}\right) \Psi\left\{\mathbf{r}_{i}\right\}=0, \quad i=1,2, \ldots, N
$$

where $\Psi\left\{\mathbf{r}_{i}\right\} \equiv \Psi\left(\mathbf{r}_{1}, \ldots, \mathbf{r}_{N}\right)$ is the antisymmetric $N$ electron wave function.

Following Ref. 3, we make a singular gauge transformation $\Psi\left\{\mathbf{r}_{i}\right\}=\exp \left[\operatorname{im} \sum_{i<j} \operatorname{Im} \ln \left(\bar{z}_{i}-\bar{z}_{j}\right)\right] \Phi\left\{\mathbf{r}_{i}\right\}$ ( $m$ is an 
odd integer) to obtain a symmetric wave function $\Phi\left\{\mathbf{r}_{i}\right\} \equiv \Phi\left(\mathbf{r}_{1}, \ldots, \mathbf{r}_{N}\right)$. The constraint equation (2) is then expressed as

$$
\left(\widetilde{\Pi}_{i}^{x}-i \widetilde{\Pi}_{i}^{y}\right) \Phi\left\{\mathbf{r}_{i}\right\}=0, \quad i=1,2, \ldots, N
$$

with $\widetilde{\mathbf{\Pi}}=\boldsymbol{\Pi}+\mathbf{a}(\mathbf{r})$. And $\mathbf{a}(\mathbf{r})$ is a statistical gauge field ${ }^{10}$ defined by

$$
a^{\alpha}\left(\mathbf{r}_{i}\right)=m \epsilon^{\alpha \beta} \sum_{j \neq i} \frac{r_{i}^{\beta}-r_{j}^{\beta}}{\left|\mathbf{r}_{i}-\mathbf{r}_{j}\right|^{2}}
$$

In order to apply the Chern-Simons gauge-theory approach, we express the constraint equation in the form of a field-theory equation by using the second quantized formalism. As usual we introduce a quantum-field operator $\psi(\mathbf{r})$ and its conjugate $\psi^{\dagger}(\mathbf{r})$, which satisfy the Bose commutation relations. We then construct a symmetric basis vector

$$
\left|\left\{\mathbf{r}_{i}\right\}\right\rangle=(N !)^{1 / 2} \psi^{\dagger}\left(\mathbf{r}_{1}\right) \cdots \psi^{\dagger}\left(\mathbf{r}_{N}\right)|0\rangle
$$

and define $|\Phi\rangle$ such that the symmetric wave function $\Phi\left\{\mathbf{r}_{i}\right\}$ is obtained by $\left\langle\left\{\mathbf{r}_{i}\right\} \mid \Phi\right\rangle$. Then, the constraint equation (3) is expressed as

$$
\left(\widetilde{\Pi}^{x}-i \widetilde{\Pi}^{y}\right) \psi(\mathbf{r})|\Phi\rangle=0,
$$

where $\widetilde{\mathbf{\Pi}}(\mathbf{r})=\mathbf{\Pi}+\widetilde{a}(\mathbf{r})$

$$
\widetilde{a}^{\alpha}(\mathbf{r})=m \epsilon^{\alpha \beta} \int d \mathbf{r}^{\prime} \frac{r^{\beta}-r^{\prime \beta}}{\left|\mathbf{r}-\mathbf{r}^{\prime}\right|^{2}} \psi^{\dagger}\left(\mathbf{r}^{\prime}\right) \psi\left(\mathbf{r}^{\prime}\right) .
$$

At this point we should remark that if we disregard the external magnetic field and the operator nature of equation (5) it is formally the same as Jackiw and Pi's self-dual equation, ${ }^{11}$ although the physics is quite different.

Since $\Phi\left\{\mathbf{r}_{i}\right\}$ is a symmetric wave function, we may apply the collective-field method ${ }^{7}$ to this problem. In what follows we closely follow the most recent formulation. ${ }^{8}$ The essence of the method is that one first regards the symmetric wave function as a functional of the particle density function, namely,

$$
\Phi\left\{\mathbf{r}_{i}\right\}=\left.\Phi[\rho(\mathbf{r})]\right|_{\rho(\mathbf{r})=\sum_{i} \delta\left(\mathbf{r}-\mathbf{r}_{i}\right)},
$$

and convert equations expressed in terms of particle coordinates [in our case Eq. (3)] into functional differential equations of collective field, $\rho(\mathbf{r})$. Let us first note

$$
\begin{aligned}
|\Phi\rangle & =\int \cdots \int \Pi d \mathbf{r}_{i} \Phi\left\{\mathbf{r}_{i}\right\}\left|\left\{\mathbf{r}_{i}\right\}\right\rangle \\
& =\Phi[\hat{\rho}] N !^{-1 / 2}\left[\int d \mathbf{r} \psi^{\dagger}(\mathbf{r})\right]^{N}|0\rangle,
\end{aligned}
$$

where $\hat{\rho}(\mathbf{r})=\psi^{\dagger}(\mathbf{r}) \psi(\mathbf{r})$ is the density operator and $\hat{\rho}(\mathbf{r})\left|\left\{\mathbf{r}_{i}\right\}\right\rangle=\sum_{i} \delta\left(\mathbf{r}-\mathbf{r}_{i}\right)\left|\left\{\mathbf{r}_{i}\right\}\right\rangle$. We multiply (5) by $\psi^{\dagger}(\mathbf{r})$ and express it in terms of charge and current density, $\hat{\rho}(\mathbf{r})$ and $\hat{\mathbf{j}}(\mathbf{r})=i \psi^{\dagger}(\mathbf{r}) \nabla \psi(\mathbf{r})$. Using the commutation relations among $\rho$ 's and $j$ 's and noticing

$$
\widehat{\mathbf{j}}(\mathbf{r}) N !^{-1 / 2}\left[\int d \mathbf{r} \psi^{\dagger}(\mathbf{r})\right]^{N}|0\rangle=0,
$$

we obtain the collective-field representation of the constraint equation as

$$
\left[\frac{\partial}{\partial z} \frac{\delta}{\rho(\mathbf{r})}+\frac{i}{\hbar}\left(\frac{e}{c} A(\mathbf{r})+a(\mathbf{r})\right] \Phi[\rho]=0,\right.
$$

where $A=\left(A_{x}-i A_{y}\right) / \sqrt{2}, \quad a=\left(a_{x}-i a_{y}\right) / \sqrt{2} \quad$ with $A_{x}, A_{y}, a_{x}, a_{y}$ satisfying $\boldsymbol{\nabla} \times \mathbf{A}=\boldsymbol{B}$ and $\boldsymbol{\nabla} \times \mathbf{a}=2 \pi m \rho$, respectively. In the symmetric gauge the constraint equation is

$$
\left(\frac{\partial}{\partial z} \frac{\delta}{\delta \rho(\mathbf{r})}+\frac{1}{2 \lambda^{2}} \bar{z}-\frac{m}{2} \int d \mathbf{r}^{\prime} \frac{1}{z-z^{\prime}} \rho(\mathbf{r})\right) \Phi[\rho]=0,
$$

which can be solved as $\Phi[\rho]=\Phi_{m}[\rho] \varphi[\rho]$ with $\partial\{\delta \varphi[\rho] / \delta \rho(\mathbf{r})\} / \partial z=0$ and

$$
\begin{aligned}
\Phi_{m}[\rho]=\exp [ & -\frac{1}{2 \lambda^{2}} \int d \mathbf{r}|z|^{2} \rho(\mathbf{r}) \\
& \left.+\frac{m}{2} \int d \mathbf{r} d \mathbf{r}^{\prime} \rho(\mathbf{r}) \ln \left|z-z^{\prime}\right| \rho\left(\mathbf{r}^{\prime}\right)\right] .
\end{aligned}
$$

As we can see from the derivation, the collective-field equations such as (8) and (9) are the operator equations, which are to be operated on to

$$
N !^{-1 / 2}\left[\int d \mathbf{r} \psi^{\dagger}(\mathbf{r})\right]^{N}|0\rangle \text {. }
$$

Or, equivalently, at the end of the calculation, $\rho(\mathbf{r})$ in the equation should be replaced by $\Sigma_{i} \delta\left(\mathbf{r}-\mathbf{r}_{i}\right)$. If we do that, combining with the singular gauge transformation which connects $\Phi\left(\mathbf{r}_{i}\right)$ with $\Psi\left(\mathbf{r}_{i}\right)$, we note that (10) corresponds exactly Laughlin's wave function.

Introducing then $\Phi[\rho]=R[\rho] \exp (i \Theta[\rho])$ with $R[\rho]$ and $\Theta[\rho]$ both as real functionals, from the constraint equation (9) we obtain

$\nabla^{2} \frac{\delta \ln R[\rho]}{\delta \rho(\mathbf{r})}+\frac{1}{\lambda^{2}}-2 \pi m \rho(\mathbf{r})=-\epsilon_{i j} \partial_{i} \partial_{j} \frac{\delta \Theta[\rho]}{\delta \rho(\mathbf{r})}$

and the equations which we assume to be automatically satisfied, i.e., $\nabla^{2} \delta \Theta[\rho] / \delta \rho(\mathbf{r})=0$ and $\epsilon_{i j} \partial_{i} \partial_{j} \delta \ln R[\rho] /$ $\delta \rho(\mathbf{r})=0$. The first term of Eq. (11), contributed by the modulus part of the wave functional, plays a nontrivial role which bears some subtle effects at the microscopic level. If we set $R[\rho]=\Phi_{m}[\rho] R^{\prime}[\rho]$ with $\Phi_{m}[\rho]$ given by Eq. (10), from the constraint equation $\partial\{\delta \varphi[\rho] /$ $\delta \rho(\mathbf{r})\} / \partial z=0$, we obtain

$$
\nabla^{2} \frac{\delta \ln R^{\prime}[\rho]}{\delta \rho(\mathbf{r})}=-\epsilon_{i j} \partial_{i} \partial_{j} \frac{\delta \Theta[\rho]}{\delta \rho(\mathbf{r})} .
$$

It is straightforward to verify that if we require the right-hand side of Eq. (12) to be $2 \pi \delta(r)$, up to a solution of the homogeneous part of the left-hand side of Eq. (12), we could have a solution like

$$
\begin{aligned}
& R^{\prime}[\rho]=\exp \left[\int d \mathbf{r} \rho(\mathbf{r}) \ln |\bar{z}|\right), \\
& \Theta[\rho]=\int d \mathbf{r} \rho(\mathbf{r}) \operatorname{Im} \ln \bar{z},
\end{aligned}
$$

which corresponds precisely to Laughlin's quasihole wave function $\Pi_{i} \bar{z}_{i}$.

Now let us obtain an equation for the density distribution $\langle\rho(\mathbf{r})\rangle$ where 


$$
\begin{aligned}
\langle(\cdots)\rangle & =\frac{\langle\Phi|(\cdots)| \Phi\rangle}{\langle\Phi \mid \Phi\rangle} \\
& =\frac{\int \cdots \int D \rho J[\rho] \Phi^{\dagger}[\rho](\cdots) \Phi[\rho]}{\int \cdots \int D \rho J[\rho]|\Phi[\rho]|^{2}}
\end{aligned}
$$

and $J[\rho]$ is the Jacobian, which is given in the leading order of $1 / N$ expansion by ${ }^{7,8}$

$$
\begin{aligned}
J[\rho]= & \delta\left[\int d \mathbf{r} \rho(\mathbf{r})-N\right] \\
& \times \exp \left[-\int d \mathbf{r} \rho(\mathbf{r}) \ln [\rho(\mathbf{r}) / \bar{\rho}]\right] .
\end{aligned}
$$

Inserting the operator relation (9), or equivalently (11), into $\cdots$ in (13) we obtain

$$
\frac{1}{2} \nabla^{2}\langle\ln \rho(\mathbf{r})\rangle+\frac{1}{\lambda^{2}}-2 \pi m\langle\rho(\mathbf{r})\rangle=2 \pi \rho_{V}(\mathbf{r}),
$$

where $2 \pi \rho_{V}(\mathbf{r})=-\epsilon_{i j} \partial_{i} \partial_{j} \vartheta_{s}(\mathbf{r})$ with $\vartheta_{s}(\mathbf{r})$ being defined as $\langle\delta \Theta[\rho] / \delta \rho(\mathbf{r})\rangle$, and $\rho_{V}(\mathbf{r})$ can be interpreted as the density of vortices. In the mean-field approximation, the first term $\langle\ln \rho(\mathbf{r})\rangle$ can be approximated as $\ln \langle\rho(\mathbf{r})\rangle$ and Eq. (15) becomes a differential equation for the particle distribution provided the right-hand side of (15) is independent of $\rho$.

Taking the spatial average for Eq. (15) and noticing that the log term will not contribute even when $\rho(\mathbf{r})$ has isolated zeroes, we obtain $1 / 2 \pi \lambda^{2}-m \bar{\rho}-\bar{\rho}_{V}=0$ with $\bar{\rho}$ and $\bar{\rho}_{V}$ being the average density of electrons and vortices, respectively. This is in fact one of the next hierarchy equations. For a FQHE system of zero vorticity, $\rho_{V}=0$, we have immediately the well-known quantization condition for the $\bar{\rho}$ and $\bar{\rho}=1 / 2 \pi m \lambda^{2}$, accordingly $v=1 / m .^{5}$ For a system of a single quasihole vortex centered at the origin, $\int d \mathbf{r}\left[\rho(\mathbf{r})-\left(2 \pi m \lambda^{2}\right)^{-1}\right]=1 / m$ means that the single vortex has $1 / m$ electric charge. From the discussion of the quasihole solution for Eq. (12) we can easily verify that Laughlin's quasihole has a vortex density $\rho_{V}(\mathbf{r})=\delta(\mathbf{r})$. Because the prefactor of the Laughlin's quasiparticle wave function has its phase part conjugate to that of the quasihole, we anticipate that it will give rise to a vortex density $\rho_{V}(\mathbf{r})=-\delta(\mathbf{r})$. Based on the above observations we interpret Eq. (15) as the vortex equation for the quasiparticles that is derived directly from the microscopic theory without referring to the phenomenological Ginzberg-Landau approach for the FQHE. ${ }^{4-6}$

Since $\rho$ and $\vartheta$ are canonically conjugate to each other, for systems without vortex singularities, a Fourier transformation can be introduced: $\Phi[\rho]$ $=\int \mathscr{D} \vartheta \exp \left[i \int d \mathbf{r} \rho(\mathbf{r}) \vartheta(\mathbf{r})\right] \widetilde{\Phi}[\vartheta]$. It is also straightforward to show that the Laughlin-like wave functional $\Phi_{m}[\rho]$, Eq. (10), has its $\vartheta$ representation as

$$
\widetilde{\Phi}_{m}[\vartheta]=\exp \left[\frac{1}{2 \pi m}\left[\frac{1}{2} \int d \mathbf{r} \vartheta(\mathbf{r}) \nabla^{2} \vartheta(\mathbf{r})-i \lambda^{2} \int d \mathbf{r} \vartheta(\mathbf{r})\right]\right]
$$

But for systems with vortexlike singular behavior, in addition to the regular $\vartheta$ particle, we should introduce the degrees of freedom for the vortices: $\rho_{B}(\mathbf{r})$ : Therefore we generalize (16) as

$$
\begin{aligned}
\Phi[\rho]= & \int \mathcal{D} \vartheta \mathcal{D} \rho_{V} \exp \left[i \int d \mathbf{r} \rho(\mathbf{r})\left\{\vartheta(\mathbf{r})+\vartheta_{s}\left[\mathbf{r}, \rho_{V}\right]\right\}\right) \\
& \times \widetilde{\Phi}\left[\rho_{V} ; \vartheta\right]
\end{aligned}
$$

where the $\vartheta_{s}-\rho_{V}$ functional dependence is set as $\epsilon_{i j} \partial_{i} \partial_{j} \vartheta_{s}\left[\mathbf{r} ; \rho_{V}\right]=-2 \pi \rho_{V}$ or its inverse $\vartheta_{s}\left[\mathbf{r} ; \rho_{V}\right]$ $=\int d \mathbf{r}^{\prime} \operatorname{Im} \ln \left(\bar{z}-\bar{z}^{\prime}\right)$, which is fixed up to an additive regular function [independent of $\rho_{V}(\mathbf{r})$ ]. We call (17) a generalized duality transformation, since the electron degrees of freedom are entirely expressed in terms of those of vortices which are dual to the electrons. Now we apply the generalized duality transformation (17) to the constraint equation of Eq. (9). Using the derivative relation, which follows straightforwardly from $\vartheta_{s}\left[\mathbf{r} ; \rho_{V}\right]$ $-\rho_{V}(\mathbf{r})$ relation introduced above,

$$
\frac{\partial}{\partial z} \frac{\delta}{\delta \rho_{V}(\mathbf{r})}=-\frac{1}{2 i} \int d \mathbf{r}^{\prime} \frac{1}{z-z^{\prime}} \frac{\delta}{\delta \vartheta_{s}\left[\mathbf{r}^{\prime}, \rho_{V}\right]}
$$

we derive

$$
\begin{aligned}
\int \frac{\partial}{\partial z} \frac{\delta}{\delta \rho_{V}(\mathbf{r})}+\frac{1}{2 \lambda_{V}^{2}} \bar{z} & -\frac{m_{V}}{2} \int d \mathbf{r}^{\prime} \frac{1}{z-z^{\prime}} \rho_{V}(\mathbf{r}) \\
& \left.+i m_{V} \frac{\partial \vartheta(\mathbf{r})}{\partial z}\right) \widetilde{\Phi}\left[\vartheta, \rho_{V}\right]=0,
\end{aligned}
$$

where $\lambda_{V}^{2}=\hbar c / e_{V} B, e_{V}=e / m$ and $m_{V}=1 / m$. We compare Eq. (19) with Eqs. (9) and (8). The first term of Eq. (19) is the gradient of a phase variable which is conjugate to the vortex density $\rho_{V}(\mathbf{r})$ with its singular part being connected to the "next hierarchy vorticity" for the vortex liquid. The second term describes the vortices moving in the applied magnetic field with a fractional charge $e_{V}=e / m$, while the third term is the contribution of statistical gauge field induced by vortices themselves with a statistic index $m_{V}=1 / m$. Setting $\rho_{V}(\mathbf{r})=\sum_{\alpha} \delta\left(\mathbf{r}-\mathbf{R}_{\alpha}\right)$, with $\mathbf{R}_{\alpha}$ being the coordinates of the vortices, and reversing the "bosonization" procedure, we see that the vortices obey the $m_{V}=1 / m$ fractional statistics.

We can make one more "singular" gauge transformations with an even integer statistics index by simply substituting $m_{V}+2 p$ for $m_{V}$ ( $p$ integers) in the third term of Eq. (19). It exhausts all possibilities that preserve the same statistics for the vortices. Applying the same procedure, by which we derived $1 / 2 \pi \lambda^{2}-m \bar{\rho}-\bar{\rho}_{V}=0$, to the constraint Eq. (19) we obtain $1 / 2 \pi \lambda_{V}-\left(m_{V}+2 p\right) \bar{\rho}_{V}$ $=0$. From the above two expressions we obtain the filling factor as $v=1 /\left[m+(2 p)^{-1}\right]$. It is quite clear by now that by successively applying this procedure we can derive the hierarchy scheme for the filling factors in a natural way. 
One of the authors (Z.B.S.) is grateful to Dr. D. H. Lee for a stimulating discussion. The research of B. S. was supported in part by NSF Grant No. PHY-9020495. D.N.S. and Z.B.S. acknowledge the warm hospitality they received from the TCSUH, the partial support by
Grant No. MDA 972-88.G-002 from U.S. Defense Advanced Research Projects Agency and the state of Texas and NSF Grant No. 8714455 and NSFC Grant No. 9187008-02.
${ }^{1}$ See, e.g., The Quantum Hall Effect, 2nd ed., edited by R. E. Prange and S. M. Girvin (Springer-Verlag, Berlin, 1989).

${ }^{2}$ R. B. Laughlin, Phys. Rev. Lett. 50, 1385 (1983).

${ }^{3}$ S. M. Girvin, The Quantum Hall Effect (Ref. 1), Chap. 10; S. M. Girvin and A. H. MacDonald, Phys. Rev. Lett. 58, 1252 (1987).

${ }^{4}$ N. Read, Phys. Rev. Lett. 62, 86 (1989).

${ }^{5}$ S. C. Zhang, T. H. Hanson, and S. Kivelson, Phys. Rev. Lett. 62, 980(E) (1989); Dong-Hai Lee and Shou-Cheng Zhang, Phys. Rev. Lett. 66, 1220 (1991).

${ }^{6}$ D. H. Lee and M. P. A. Fisher, Phys. Rev. Lett. 63, 903 (1989).
${ }^{7}$ A. Jevicki and B. Sakita, Nucl. Phys. B 165, 511 (1989); B. Sakita, Quantum Theory of Many Variable System and Fields (World Scientific, Singapore, 1985).

${ }^{8}$ D. Karabali and B. Sakita, Int. J. Mod. Phys. A (to be published).

${ }^{9}$ D. M. Haldane, Phys. Rev. Lett. 51, 605 (1983).

${ }^{10}$ F. Wilczek, Phys. Rev. Lett. 48, 1144 (1982); D. P. Arovas, J. R. Schrieffer, F. Wilczek, and A. Zee, Nucl. Phys. B 251, 117 (1985).

${ }^{11}$ R. Jackiw and So-Young Pi, Phys. Rev. Lett. 64, 2969 (1990). 